\title{
Bending fatigue behaviour of 17-4 PH gears produced with additive techonlogies
}

\author{
Franco Concli ${ }^{1}$, Luca Bonaiti ${ }^{2}$, Luca Cortese ${ }^{3}$, Filippo Nalli ${ }^{1}$, Francesco Rosa ${ }^{2}$, and Carlo \\ Gorla ${ }^{2}$
}

${ }^{1}$ Free University of Bolzano

${ }^{2}$ Politecnico di Milano

${ }^{3}$ University of Rome La Sapienza

May 5, 2020

\begin{abstract}
The introduction on the industrial market of additive technologies is changing the way in which parts are designed and manufactured. Within this context, designers are looking for the possibilities of producing parts via the addition of material, rather than its removal. Design of critical parts (e.g. gears and shafts) requires specic resistance data that, due to the intrinsic modernity of additive technologies, are not present in the literature. This paper presents the results of an experimental campaign aimed to determine the bending fatigue limit for gears made by 17-4 PH produced via selective laser melting. The fatigue limit, obtained by using two dierent statistical approaches, is presented here according to the ISO standard in order to compare it with those of different materials. Additional analysis have been done in order to investigate the failure origin.
\end{abstract}

\section{Hosted file}

Main.pdf available at https://authorea.com/users/295464/articles/429989-bending-fatiguebehaviour-of-17-4-ph-gears-produced-with-additive-techonlogies

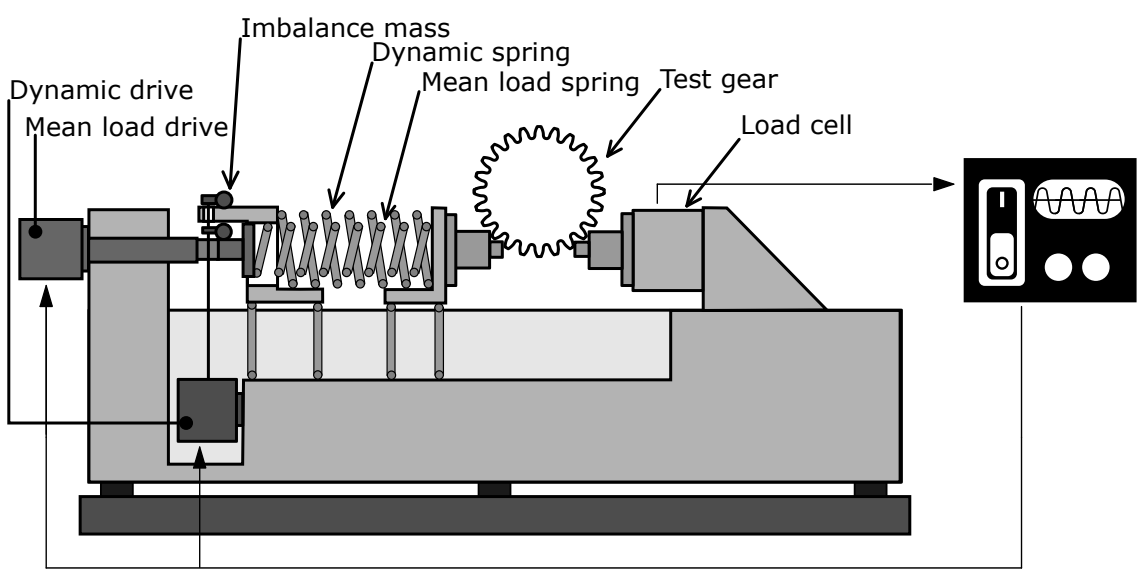



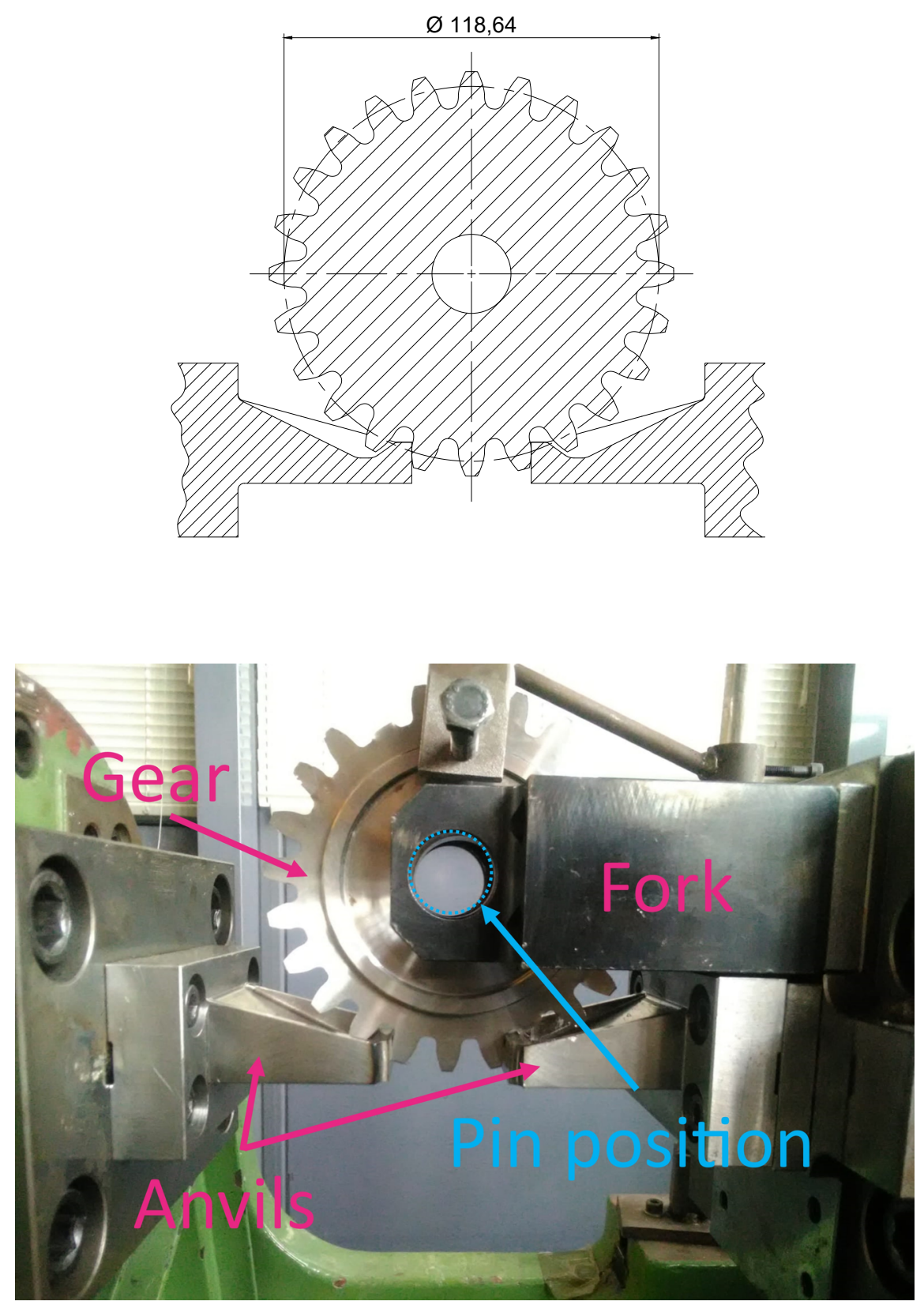

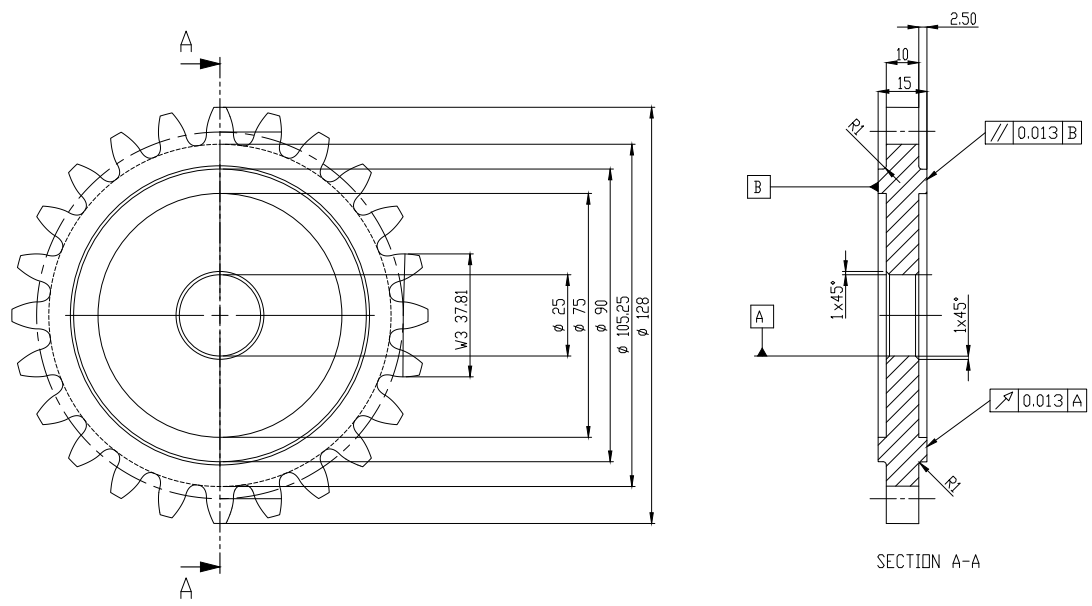

SECTIDN A-A

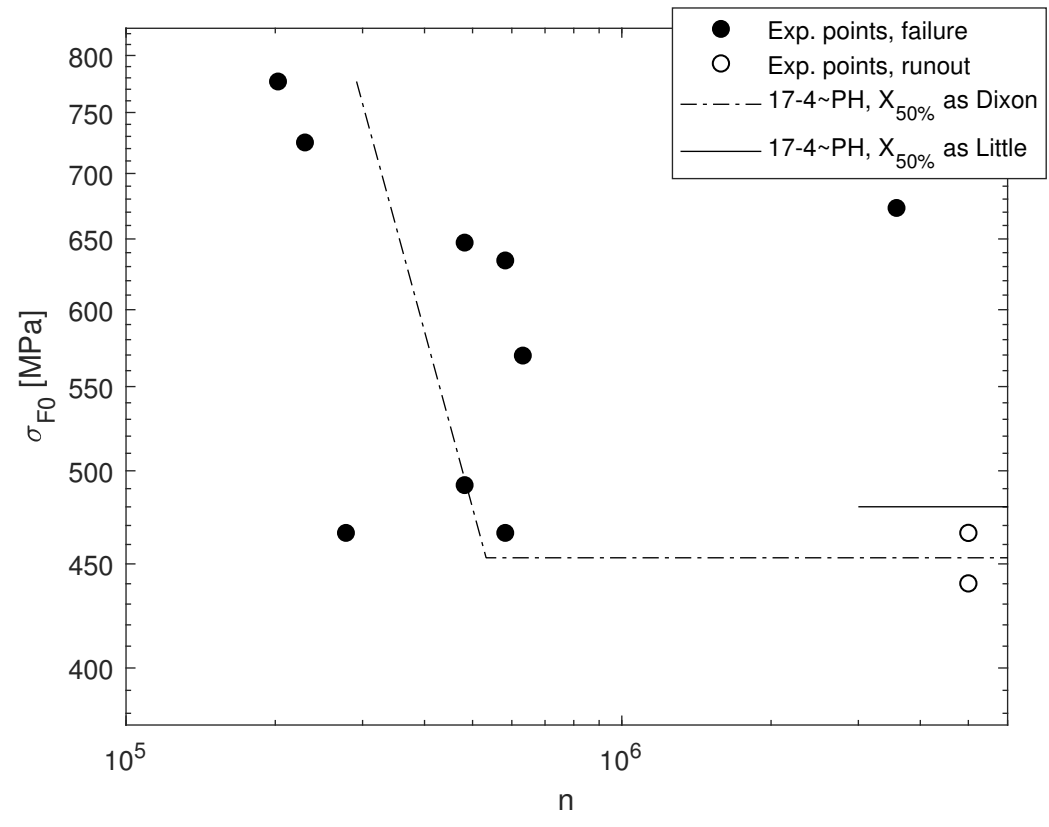




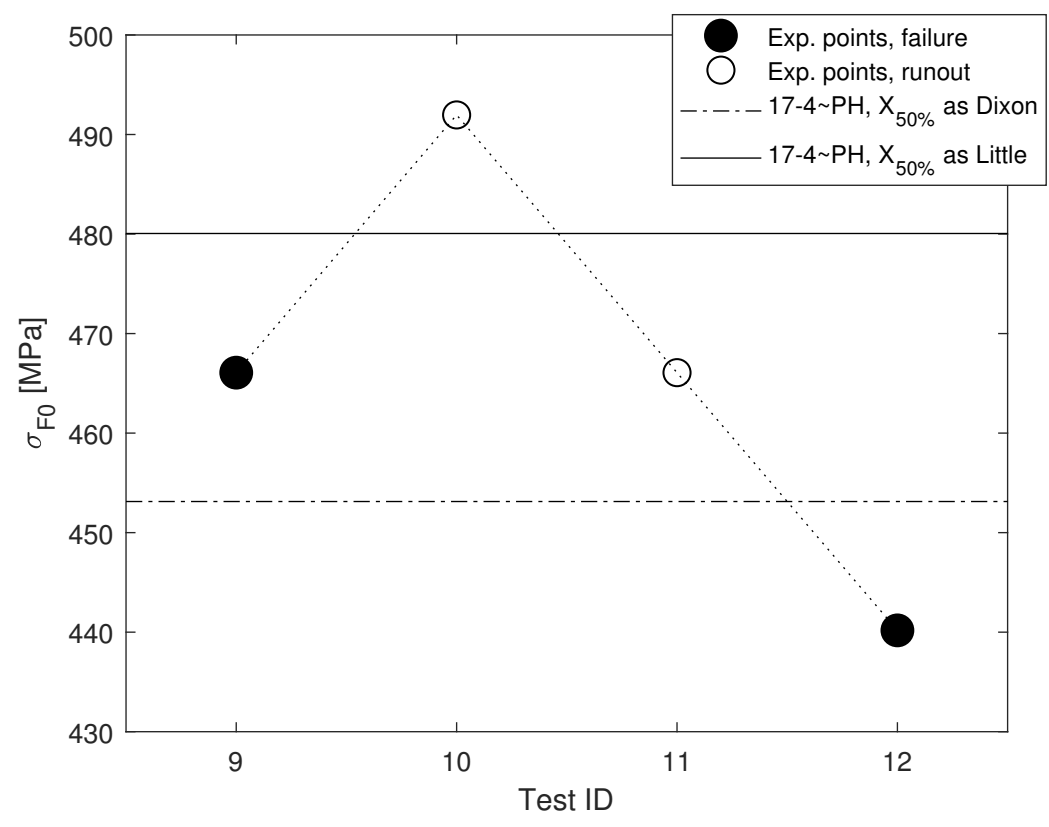




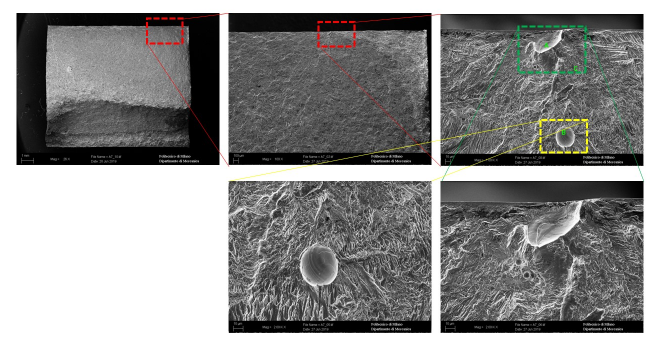

Figure 7: SEM analysis of tooth A07 


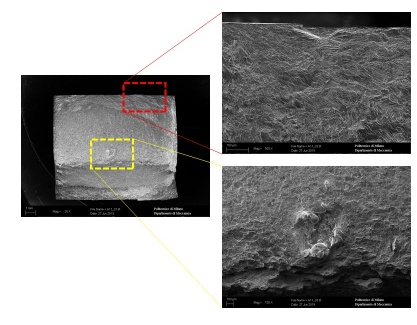

Figure 8: SEM analysis of tooth A11 


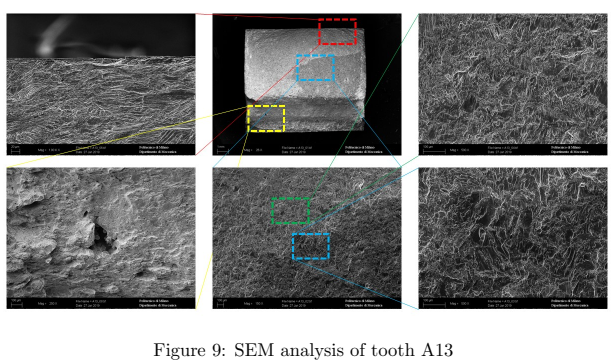

18 

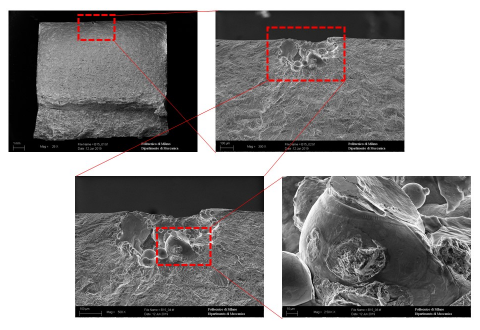

Figure 10: SEM analysis of tooth B15 


\begin{tabular}{lll} 
& & Table 1: Gear main data. \\
\hline Symbol & Value & Description \\
\hline$m_{n}$ & $5 \mathrm{~mm}$ & Normal module \\
$\alpha_{n}$ & $20^{\circ}$ & Normal Pressure angle \\
$\beta$ & $0^{\circ}$ & Pressure angle at normal section \\
$z$ & 24 & Number of teeth \\
$b$ & $10 \mathrm{~mm}$ & Facewidth \\
$x$ & -0.2 & Profile shift coefficient \\
$h_{f P}^{*}$ & 1.250 & Dedendum coefficient of the basic rack profile \\
$\rho_{f P}^{*}$ & 0.380 & Root radius factor of the basic rack profile \\
$h_{a P}^{*}$ & 1.00 & Addendum coefficient of the basic rack profile \\
\hline
\end{tabular}




\begin{aligned} & Table 2: Process parameter \\ & \hline Machine EOS M280 \\ & Laser source fiber \\ & Printing direction according to gear axis (see Fig. 4) \\ & Gas nitrogen \\ & Power $200 \mathrm{~W} \\ &$ Scanning speed $600 \mathrm{~mm} \mathrm{~s}^{-1} \\ &$ Spot diameter $100 \mathrm{~mm} \\ &$ Layer thickness $40 \mathrm{\mu m} \\ &$ Average particle size $42.61 \mathrm{~mm} \\ &$\hline\end{aligned}

21 
Table 3: Bending fatigue test results.

\begin{tabular}{ccccccc}
\hline \multicolumn{7}{c}{ Table 3: Bending fatigue test results. } \\
\hline Test ID & Gear & Tested teeth & $\begin{array}{c}F_{\min } \\
{[\mathrm{N}]}\end{array}$ & $\begin{array}{c}F_{\max } \\
{[\mathrm{N}]}\end{array}$ & Cycles & Broken tooth \\
\hline 1 & $\mathrm{~A}$ & $1-3$ & -15000 & -1500 & 202550 & 1 \\
2 & $\mathrm{~A}$ & $5-7$ & -14000 & -1400 & 229575 & 7 \\
3 & $\mathrm{~A}$ & $9-11$ & -13000 & -1300 & 3581879 & 11 \\
4 & $\mathrm{~A}$ & $13-15$ & -12500 & -1250 & 481897 & 13 \\
5 & $\mathrm{~A}$ & $17-19$ & -12250 & -1225 & 581951 & 17 \\
6 & $\mathrm{~A}$ & $21-23$ & -11000 & -1100 & 631460 & 21 \\
7 & $\mathrm{~B}$ & $1-3$ & Deleted test & & \\
8 & B & $5-7$ & -9000 & -900 & 277690 & 7 \\
9 & B & $9-11$ & -9000 & -900 & Runout & - \\
10 & B & $13-15$ & -9500 & -950 & 481897 & 15 \\
11 & B & $17-19$ & -9000 & -900 & 581951 & 19 \\
12 & B & $21-23$ & -8500 & -850 & Runout & - \\
\hline
\end{tabular}

22 


\begin{tabular}{rl} 
Table 4: Eq.(1) & parameters \\
\hline & Value \\
\hline$h_{F e}$ & $0.53 \mathrm{~mm}$ \\
$\alpha_{F e n}$ & $13.79^{\circ}$ \\
$Y_{F}$ & 1.5805 \\
$Y_{S}$ & 1.7434 \\
$Y_{\beta}, Y_{B}, Y_{D T}$ & 1 \\
\hline
\end{tabular}


Table 5: Chemical punctual analysis of tooth A07 (Fig.7). \begin{tabular}{llllll} 
Position & $\% \mathrm{Si}$ & $\% \mathrm{Cr}$ & $\% \mathrm{Fe}$ & $\% \mathrm{Ni}$ & $\% \mathrm{Cu}$ \\
\hline
\end{tabular} \begin{tabular}{llllll} 
A & 0.69 & 17.36 & 73.74 & 4.59 & 3.61 \\
B & 1.13 & 28.37 & 67.29 & 2.33 & 0.88 \\
\hline
\end{tabular} $\begin{array}{lllll}0.93 & 17.09 & 74.83 & 3.94 & 3.20\end{array}$ 